\title{
Prospective Multicenter Observational Study of Voglibose in Type 2 Diabetes-VICTORY
}

Sanjay Kalra, ${ }^{1}$ A Panneer Selvam, ${ }^{2}$ Amish V Shah, ${ }^{3}$ Kudugunti Neelaveni, ${ }^{4}$ Navneet Agrawal, ${ }^{5}$ Sambit Das, ${ }^{6}$ Vinay K Dhandhania, ${ }^{7}$ Subodh Jain, ${ }^{8}$ Pankaj Kumar Jha ${ }^{9}$ and Neha Raval ${ }^{9}$

1. Bharti Hospital, Karnal, India; 2. Aruna Diabetes Centre, Chennai, India; 3. Advanced Diabetes Centre Pvt. Ltd., Surat, India; 4. Endocare, Hyderabad, India; 5. Diabetes Obesity \& Thyroid Centre, Gwalior, India; 6. Dr Sambit's Centre of Diabetes and Endocrinology, Bhubaneswar, India; 7. Diabetes Care Centre, Ranchi, India; 8. Diabetes Care Centre, Allahabad, India; 9. Torrent Pharmaceuticals Ltd., Ahmedabad, India

$\mathrm{O}$ bjective: Despite its beneficial effect on postprandial hyperglycemia, the effect and usage of voglibose for the management of type 2 diabetes mellitus (T2DM) in routine clinical practice remains undetermined. The objective of this non-interventional study is to determine the real-world effectiveness of voglibose in terms of efficacy and safety, and the usage pattern as monotherapy or add-on treatment in patients with T2DM in India. Design: This was a 12-week, multi-center, prospective, observational study. Methods: Adults with inadequately controlled T2DM (glycated hemoglobin [HDA1c] 7.0-10.0\% despite diet, exercise and/or antidiabetic agents), treated with voglibose monotherapy or as add-on therapy, were recruited from 39 sites. The primary endpoint was change in HDA1C. Secondary endpoints were change in fasting and postprandial blood glucose (FBG and PPBG) levels and bodyweight, pattern of usage and safety assessments. Mean change in HDA1C, FBG and PPBG levels were analyzed using paired t-test with significance value of 5\%. Trial Registration: CTRI No. CTRI/2017/02/007878. Results: A total of 1,542 participants were enrolled and 1,365 completed two study visits. Mean HbA1C was $8.27 \%$ at baseline, which reduced to $7.29 \%$ at week $12(0.98 \%$ change, $p<0.001)$. At week 12, mean reductions in FBG and PPBG levels and bodyweight were $27.1 \mathrm{mg} / \mathrm{dL}(\mathrm{p}<0.001), 52.2 \mathrm{mg} / \mathrm{dL}$ $(p<0.001)$, and $1.00 \mathrm{~kg}(p=0.121)$, respectively. Most participants $(75 \%)$ received voglibose as add-on therapy and the most commonly prescribed concomitant oral antidiabetic drugs were metformin and sulfonylurea (29\%). Flatulence (0.5\%) and abdominal discomfort (0.3\%) were the most commonly reported adverse events in the study. Conclusions: Voglibose, monotherapy or add-on therapy, significantly reduced HbA1C, FBG and PPBG and was well tolerated in patients with T2DM in a real-world setting.

\section{Keywords}

Voglibose, HbA1c, hyperglycemia, diabetes, observational study

Disclosures: Sanjay Kalra, A Panneer Selvam, Amish V Shah, Kudugunti Neelaveni, Navneet Agrawal, Sambit Das, Vinay K Dhandhania and Subodh Jain have received consulting fees from Torrent Pharmaceuticals Ltd.

Pankaj Kumar Jha and Neha Raval are full-time employees of Torrent Pharmaceuticals Ltd.

Acknowledgments: The authors wish to thank the participating investigators for contributing participants' data for this study. Altaf Makwana and Ashutosh Kakkad were involved in the technical editing of the manuscript. Robin Nath and Saurabh Kumar provided administrative support for the conduct of this study. The authors also acknowledge Mediception Science Pvt Ltd. for providing writing assistance, and Devum Research for statistical analysis, which were funded by Torrent Pharmaceuticals Ltd., India.

Review Process: Double-blind peer review.

Compliance with Ethics: All ethical approvals required for the study was obtained before the start of the trial and details have been mentioned in Trial Registration: www.ctri.nic.in (CTRI/2017/02/007878). The study was approved by Sangini Hospital Ethics Committee, Ahmedabad, Gujarat. All procedures were followed in accordance with the responsible committee on human experimentation and with the Helsinki Declaration of 1975 and subsequent revisions, and informed consent was received from the patient involved in this study.

Authorship: All named authors meet the International Committee of Medical Journal Editors (ICMJE) criteria for authorship of this manuscript, take responsibility for the integrity of the work as a whole, and have given final approval for the version to be published.

Access: This article is freely accessible at touchENDOCRINOLOGY.com (OTouch Medical Media 2020.

Received: December 5, 2019

Accepted: February 18, 2020

Published Online: March 17, 2020

Citation: US Endocrinology. 2020;16(1):31-5

Corresponding Author: Pankaj Kumar Jha, Torrent Pharmaceuticals Ltd., Torrent House, Off Ashram Road, Ahmedabad 380009, India. E: pankajjha@torrentpharma.com

Support: The study was funded by Torrent Pharmaceuticals Ltd., India. Torrent Pharmaceuticals Ltd. have provided oversight on the conduct of the study, including design, collection, compilation and analysis of data. 
India, being an epicentre of diabetic population, has faced an increase in disease-prevalence of more than double over the past 15 years, and is projected to have about 123.5 million cases by the year 2040. ${ }^{1}$ In addition to achieving target glycated hemoglobin ( $\mathrm{HbA} 1 \mathrm{C})<7 \%$, intensive control of blood glucose levels is important in order to retard or arrest the development and progression of the microvascular complications of diabetes. Changes in postprandial blood glucose (PPBG) levels precede those in fasting blood glucose (FBG) and finally in HbA1C. Hence, the management of both FBG and PPBG is critical to achieving full glycemic control;' the latter often being more challenging to achieve. ${ }^{3}$

Voglibose, an alpha-glucosidase inhibitor, reduces PPBG when used alone or in combination with other antidiabetic drugs. Its efficacy and safety have been well established in multiple randomized controlled trials (RCTS), which conclude it to be an effective oral antidiabetic drug (OAD) with a good tolerability profile and cardiovascular benefits. ${ }^{4,5}$

The design of RCTs, often considered the gold standard for evaluating the safety and efficacy of therapeutic agents, necessitates strict eligibility criteria for patient inclusion, which questions the representability of the study population to that encountered in clinical practice. Real-world studies, on the other hand, demonstrate the picture of large datasets from diverse patient populations. Additionally, studies of an observational nature, wherein data have been collected over a long period, provide evaluation of the long-term safety and effectiveness of the drug in large heterogeneous populations, as well as information on utilization patterns. Thus, real-world studies have broader generalizability and are an effective tool to confirm the results of RCTs in clinical practice. ${ }^{6}$ However, there are scarce data available to demonstrate the effectiveness of voglibose in real-world settings, especially in the Indian population. Currently available literature covers a few Indian studies of voglibose in real-life settings, which include only a small number of patients from one or two centers. ${ }^{7.8}$ Hence, this non-interventional, observational study was planned with an objective to assess the real-world effectiveness of voglibose by determining its efficacy, safety and usage as monotherapy or add-on treatment in patients with type 2 diabetes mellitus (T2DM) from multiple sites in India.

\section{Methods}

VICTORY (A non-interventional, multicenter, prospective, observational study to understand usage and effect of voglibose as monotherapy or add on treatment in Indian type 2 diabetes patients) was a 12-week, prospective, observational, multicenter study conducted between October 2016 and August 2018. Participants were recruited by 39 private clinical practitioners (endocrinologists and diabetologists) from both urban and rural sites across India. Adult participants, of either sex, aged $\geq 18$ years, with inadequately controlled T2DM (HDA1C 7.0-10.0\%) despite diet, exercise and/or antidiabetic agents were included in the study. Patients with T1DM, those with history of hypoglycemia episodes or ketoacidosis, and other medical conditions that could potentially hinder the safe conduct of the study, were excluded. Even though considered safe, use of voglibose is contraindicated in patients with ketoacidosis and is also associated with reports of hypoglycemia. ${ }^{9}$ Based on these facts, we excluded patients with a history of hypoglycemic or ketoacidosis from this study. Patients participating in any other clinical trials, pregnant or lactating women and those of child-bearing potential and not using adequate contraception, were also excluded. Data of participants receiving voglibose $(0.2 \mathrm{mg}$ or $0.3 \mathrm{mg}$; either alone or as add-on with other OADs based on investigators' discretion) in routine clinical practice were collected.

The study was performed in compliance with the principles of the Declaration of Helsinki, in accordance with the International Conference of Harmonization Guideline for Good Clinical Practice, and in accordance with applicable regulatory requirements. All participants provided written informed consent. The study was registered at Clinical Trial Registry of India with registration number CTRI/2017/02/007878.

\section{Endpoints}

The primary endpoint was mean change in $\mathrm{HbA} 1 \mathrm{c}$ levels from baseline to week 12. Secondary endpoints were mean change in FBG, PPBG, and body weight from baseline to week 12; the usage patterns for voglibose (monotherapy or add-on therapy with other OADs); and adverse events (AEs) reported during the study period. AEs were classified as mild (no interference with usual activity), moderate (significant interference with usual activities), and severe (prevents usual activities).

\section{Statistical analysis}

Considering the large sample size, it was assumed that data were normally distributed according to the central limit theorem. Continuous variables were reported as mean and standard deviation (SD) and categorical variables were presented as absolute frequency and percentage. The data were analyzed, and the mean change in $\mathrm{HbA} 1 \mathrm{C}$, FBG, PPBG and weight along with SD were subjected to statistical analysis using paired t-test with a significance value of $5 \%$. The safety review was performed using intent-to-treat dataset (all participants who were recruited in the study irrespective of study completion), while efficacy analysis was performed using per protocol dataset (all participants who completed the study as per the protocol).

\section{Results}

A total of 1,542 participants were enrolled in the study; of these 1,365 participants (88.5\%) completed the study (Figure 1). The mean age and body weight of the study participants were 53.7 years (SD 11.0) and $71.4 \mathrm{~kg}$ (SD 12.8), respectively. Study population included more males $(n=826 ; 60.5 \%)$ than females $(n=539 ; 39.5 \%)$. The baseline characteristics of study participants are shown in Table 1.

\section{Efficacy}

Mean $\mathrm{HbA} 1 \mathrm{C}$ was $8.27 \%$ (SD 0.82) at baseline, and decreased to $7.29 \%$ (SD 0.97) at week 12 ( $p<0.001)$. However, differences between two dose groups (0.2 $\mathrm{mg}$ and $0.3 \mathrm{mg}$ ) were non-significant for either monotherapy $(p=0.133)$ or add-on therapy $(p=0.634)$ (Figure 2$)$. Significant $(p<0.001)$ reductions in FBG (27.1 mg/dL; from 146.2 to $119.1 \mathrm{mg} / \mathrm{dL}$ ) and PPBG (52.2 mg/dL; from 231.9 to $179.7 \mathrm{mg} / \mathrm{dL}$ ) were reported at week 12 . Reduction in FBG was significantly higher among participants who received $0.3 \mathrm{mg}$ monotherapy compared to $0.2 \mathrm{mg}$ monotherapy of voglibose, while between-dose group differences of FBG reduction for voglibose add-on therapy were non-significant. Reduction in PPBG was significantly higher $(p<0.001)$ among participants who received $0.3 \mathrm{mg}$ dose compared to $0.2 \mathrm{mg}$ dose of voglibose with either as monotherapy or add-on therapy (Figure 3). Mean reduction in bodyweight ( $1.00 \mathrm{~kg} ; \mathrm{p}=0.121$ ) was not significant at week 12 (Figure 4). 


\section{Figure 1: Participant disposition}

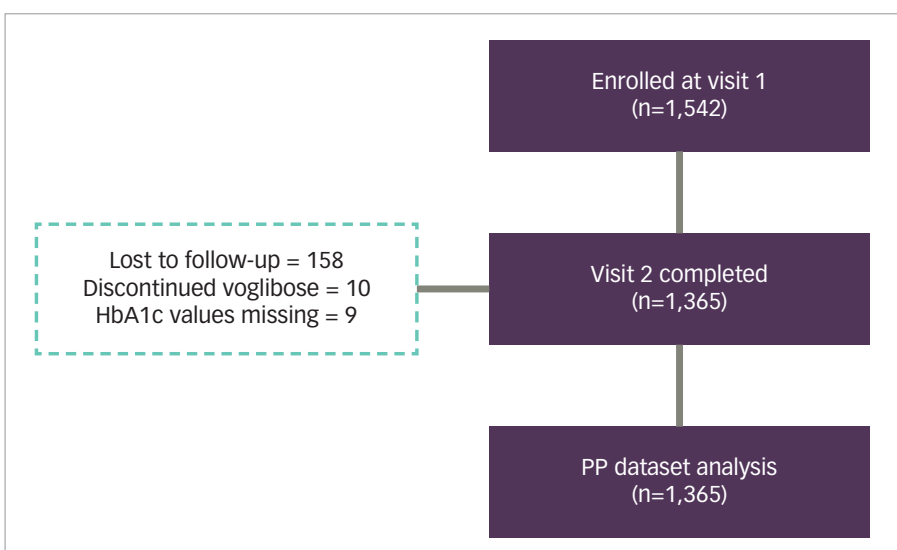

HDA1C = glycated hemoglobin; $P P=$ per protocol.

Table 1: Baseline characteristics of study participants

\begin{tabular}{|l|l|}
\hline & Overall, $\mathrm{n}=1,365$ \\
\hline Age, years & $53.7(11.0)$ \\
\hline Weight, $\mathrm{kg}$ & $71.4(12.8)$ \\
\hline Height, cm & $162.6(10.0)$ \\
\hline HbA1C, \% & $8.27(0.82)$ \\
\hline FBG, $\mathrm{mg} / \mathrm{dL}$ & $146.2(43.8)$ \\
\hline PPBG, $\mathrm{mg} / \mathrm{dL}$ & $231.9(59.4)$ \\
\hline
\end{tabular}

All data are presented as mean (standard deviation).

$F B G=$ fasting blood glucose; $H B A 1 C=$ glycated hemoglobin; $P P B G=$ postprandial blood glucose.

Figure 2: Changes in glycated hemoglobin levels with monotherapy and add-on therapy with voglibose

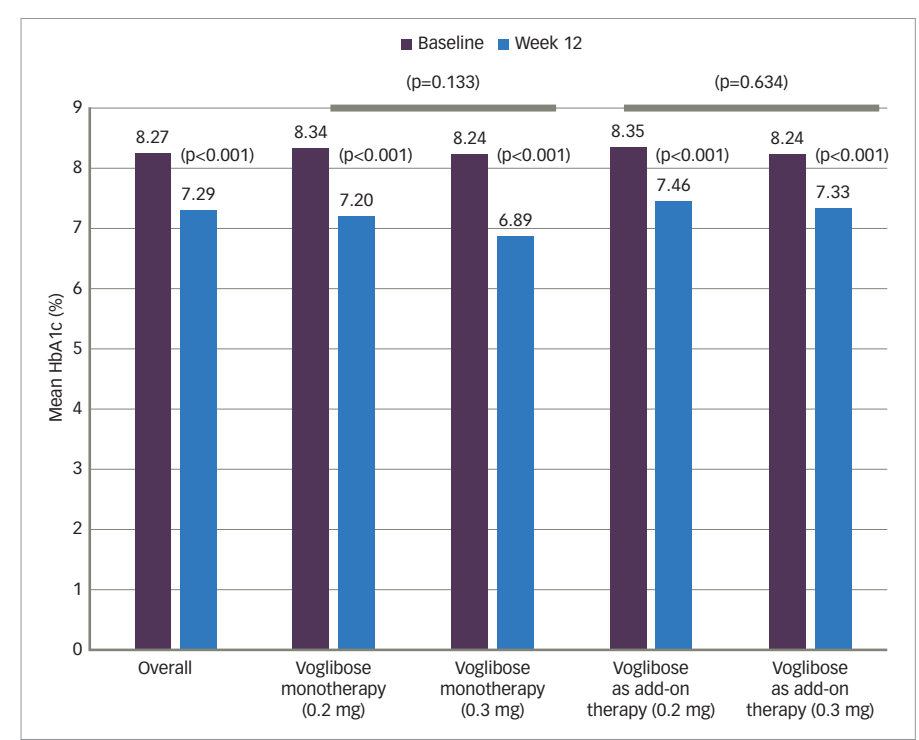

$P$ value derived using paired $t$-test for within group differences was $p<0.001$ for both doses at week 12.

$H \mathrm{BA} 1 \mathrm{C}=$ glycated hemoglobin.
Figure 3: Mean change in fasting blood glucose (A) and postprandial blood glucose (B) from baseline to week 12

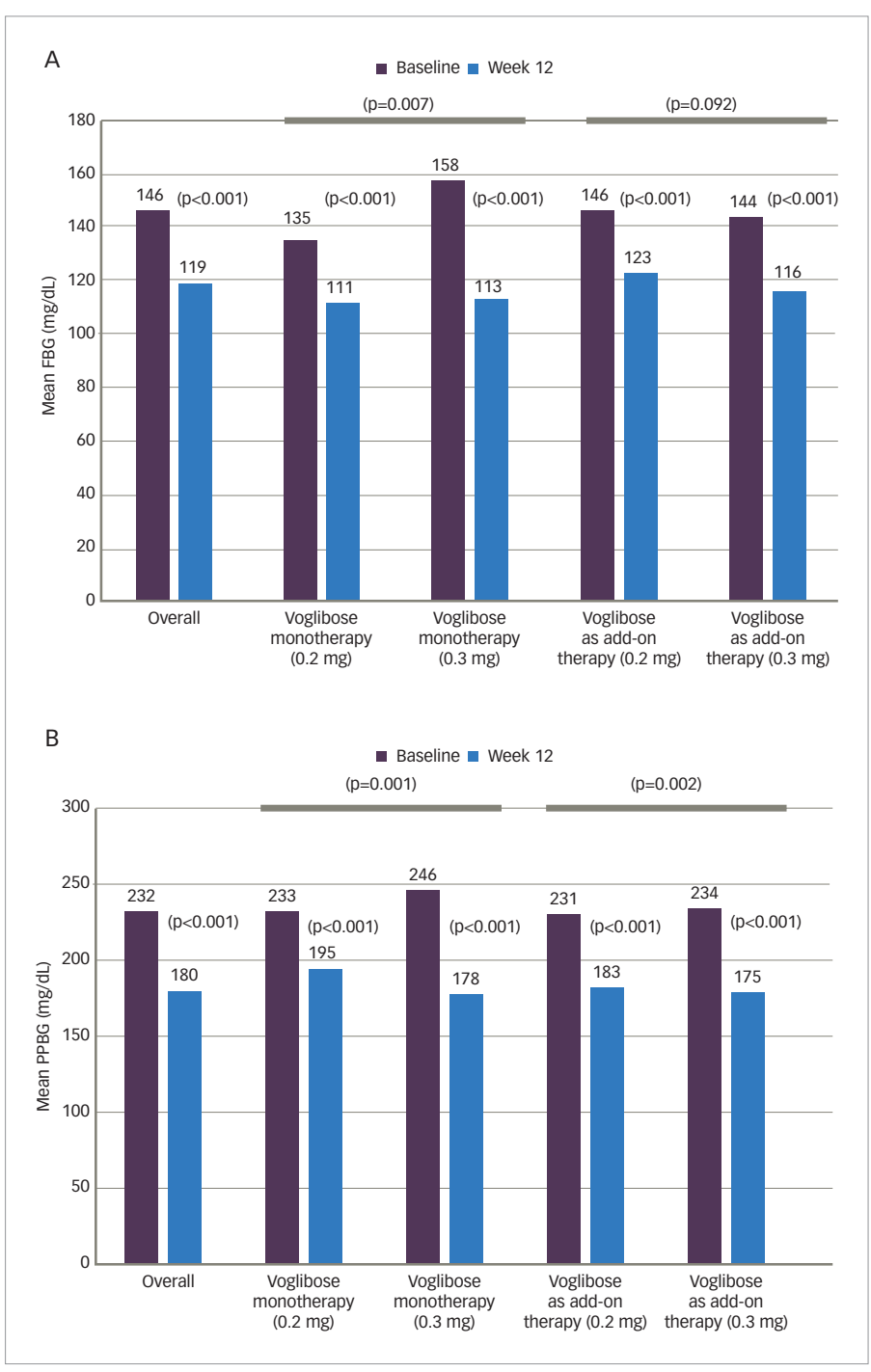

$P$ value derived using paired $t$-test for within group differences was $p<0.001$ for both doses at week 12; $p$ value derived using independent sample t-test for between group differences was $p=0.007$ and $p=0.092$ for $F B G$, and $p=0.001$ and $p=0.002$ for $P P B G$ with monotherapy and add-on therapy, respectively.

$F B G=$ fasting blood glucose; $H D A 1 C=$ glycated hemoglobin; $P P B G=$ postprandial blood glucose.

\section{Safety}

The AEs reported during this study were flatulence $(n=7 ; 0.5 \%)$, abdominal discomfort $(n=4 ; 0.3 \%)$, gastritis $(n=2 ; 0.1 \%)$, diarrhea $(n=2 ; 0.1 \%)$ and leg pain $(n=1 ; 0.1 \%$ ) (Table 2). Most of the AEs were mild $(n=14)$, and two were moderate. No serious AE or hypoglycemia episode was reported in any participant. AEs were managed by the investigators as per their routine clinical practice.

\section{Usage pattern}

A total of 345 (25.3\%) participants received voglibose as monotherapy, while $1,020(74.7 \%)$ received it as add-on therapy to other antidiabetic drugs. Of those who received voglibose as add-on therapy, $12.4 \%$ 
Figure 4: Mean change in bodyweight from baseline to week 12

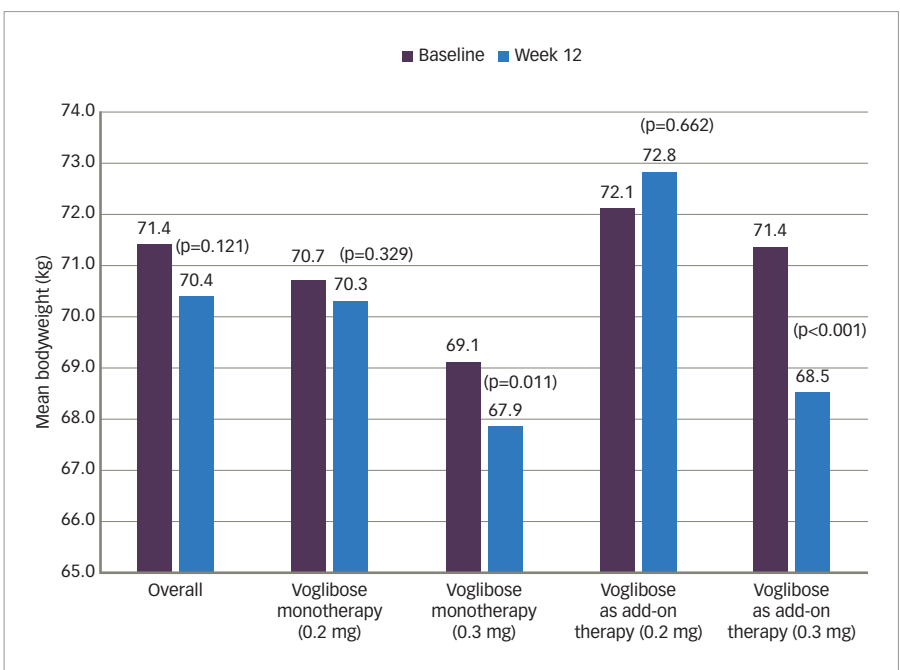

There was no significant change in bodyweight with voglibose monotherapy. When used as add-on therapy, $0.3 \mathrm{mg}$ of voglibose resulted in significant reduction in bodyweight $(p<0.001)$

received it as part of dual drug therapy, $36.6 \%$ as triple-drug therapy, $19.3 \%$ as quadruple-drug therapy, $4.4 \%$ as five-drug therapy, and $2.0 \%$ as other combinations. A total of $393(28.8 \%)$ participants received voglibose as add-on with metformin and sulfonylurea; 176 (12.9\%) with metformin, sulfonylurea and dipeptidyl peptidase 4 inhibitors (DDP4-I); 102 (7.5\%) with only metformin; 70 (5.1\%) with metformin and DDP4-I; and 47 (3.4\%) with only sulfonylurea. A total of 232 (17.0\%) participants received voglibose as part of other antidiabetic combinations that included various permutations with sulfonylureas, metformin, DDP4-I, and insulin, as well as thiazolidinediones.

\section{Discussion}

Alpha-glucosidase inhibitors (voglibose and acarbose) were introduced as first-line therapy to attain better PPBG control by the Research Society for the Study of Diabetes in India diabetes management guidelines. ${ }^{10}$ Despite being reported as an efficacious and safe treatment option, the utilization of voglibose remains undetermined for the management of diabetes in real-life settings. The VICTORY study includes analysis of a large population of patients with T2DM from varied geographical regions in India.

Most participants (74.7\%) in our study were taking multiple antidiabetic agents. Voglibose was most commonly used as add-on to metformin and sulfonylureas (28.8\%) - a finding seen in several clinical trials of patients with T2DM treated with voglibose as add-on therapy. ${ }^{11-3}$ Twelve-week treatment with voglibose resulted in significant reduction in HbA1c $(0.98 \%, p<0.001)$. Similar reductions in HbA1c with monotherapy or add-on therapy with voglibose have been reported in randomized studies. In a 6-month, single-blind, randomized, prospective study that compared voglibose with miglitol and acarbose, voglibose $(0.2 \mathrm{mg}$ three times daily for 1 month followed by $0.3 \mathrm{mg}$ three times daily for 3 months) significantly reduced HbA1c by $1.57 \% .{ }^{14}$ Jindal et al. ( $n=30$; HbA1c $\left.7-10 \%\right)$ reported reduction in mean $\mathrm{HbA} 1 \mathrm{c}$ of $1.96 \%$ with voglibose $(0.2 \mathrm{mg}$ three times daily) when added to glimepiride ( $2 \mathrm{mg}$ twice daily) and metformin
Table 2: Adverse events observed during study

\begin{tabular}{|l|l|l|}
\hline & & \\
\hline Adverse event & $n$ & $\%$ \\
\hline Flatulence & 7 & $0.5 \%$ \\
\hline Abdominal discomfort & 4 & $0.3 \%$ \\
\hline Gastritis & 2 & $0.1 \%$ \\
\hline Diarrhea & 2 & $0.1 \%$ \\
\hline Leg pain & 1 & $0.1 \%$ \\
\hline
\end{tabular}

$N=1,542$

(500 mg twice daily). ${ }^{15}$ In a retrospective study, overweight or obese Indian participants with diabetes that was inadequately controlled with metformin and sulfonylureas (body mass index $\geq 25 \mathrm{~kg} / \mathrm{m}^{2}$; HbA1c level $>8.0-9.5 \%$ ), received voglibose ( $n=41 ; 0.2$ or $0.3 \mathrm{mg}$ three times daily) or acarbose $(n=36)$. Voglibose showed significant reduction in HbA1c level $(0.32 \%)$ at 8 weeks $(8.61 \pm 1.24$ versus $8.29 \pm 1.3 ; p<0.05)$ and at 12 weeks $(8.61 \pm 1.24$ versus $7.53 \pm 1.25 ; p<0.01) .^{16}$

Mean reduction of $27.1 \mathrm{mg} / \mathrm{dL}$ in FBG was observed in our study. Similar reductions of $20.2 \mathrm{mg} / \mathrm{dL}$ and $21.4 \mathrm{mg} / \mathrm{dL}$ have been reported by Rao et al. ${ }^{17}$ and Ismail et al., ${ }^{14}$ respectively. On the other hand, Talaviya et al. ${ }^{16}$ and Jindal et al. ${ }^{15}$ have reported FBG reductions of $8.2 \mathrm{mg} / \mathrm{dL}$ and $14.7 \mathrm{mg} / \mathrm{dL}$, respectively. We observed a mean reduction of $52.2 \mathrm{mg} / \mathrm{dL}$ in PPBG. This is similar to reductions of $64.4 \mathrm{mg} / \mathrm{dL}$ and $71.3 \mathrm{mg} / \mathrm{dL}$ reported by Rao et al. ${ }^{17}$ and Jindal et al., ${ }^{15}$ respectively, while it is higher than the reported reduction of $24.9 \mathrm{mg} / \mathrm{dL}$ in the study by Talaviya et al. ${ }^{16}$ In the present study, significantly higher reduction of FBG and PPBG was observed among participants on voglibose $0.3 \mathrm{mg}$ dose compared to $0.2 \mathrm{mg}$ dose, except for FBG reduction in add-on therapy group. The reductions in PPBG with voglibose suggests it may have a major role in the achievement of desired HbA1c. Reductions in PPBG have been shown to significantly reduce HbA1c levels in participants with T2DM. ${ }^{18}$

In the present study, there was a non-significant $(p=0.121)$ reduction of $1.0 \mathrm{~kg}$ in bodyweight observed at end of 12 weeks. Contrary to our observations, Talaviya et al. reported significant reduction in body weight at 8 weeks $(77.85 \pm 9.43$ versus $74.84 \pm 8.1 ; p<0.05)$ and 12 weeks $(77.85$ \pm 9.43 versus $73.52 \pm 7.68 ; p<0.05)$ of treatment with voglibose when compared to baseline. ${ }^{16}$ However, Jindal et al. have reported no significant change in body weight after 6 months of treatment with voglibose. ${ }^{15}$ Differences seen between the results of this study and that of the ones by Talaviya et al. ${ }^{16}$ could be explained by the retrospective nature, obese patient population, and small sample size $(n=77)$ of the latter.

In our experience, voglibose monotherapy or add-on therapy was safe for use in participants with T2DM, with no serious AEs reported. Flatulence and abdominal discomfort were the most commonly reported AEs and most AEs were of mild intensity. This is consistent with the safety and tolerability of voglibose reported in several studies. ${ }^{14,17,16,19-21}$ However, the under-reporting of safety events, as suggested by the low rates of $A E s$, is a common problem in observational studies.7,17,22 In contrast to RCTs, the data collection of AEs was based on voluntary reporting by the investigators, who are more likely to report serious AEs in clinical practice. 
The short observation period and the lack of a control group are the main limitations of this study. However, the latter increases the propensity of the results to be more generalizable and replicable in clinical practice, which is necessary to complement the information retrieved from RCTs. Real-life studies and RCTs both have strengths and limitations, and those should be appreciated as complementary to one another.

The strength of the VICTORY study is the large sample size from multiple real-life set-ups across India. This clearly supports widespread use of voglibose in patients with T2DM in real-world practice. Additionally, it also indicates the enthusiasm and interest of both patients and healthcare professionals to be part of such undertakings. Having excluded the non-qualifying and incomplete data, we tried to optimize the quality of the data, which were utilized for the final analysis in this study.

\section{Conclusions}

The VICTORY study demonstrates the real-life effectiveness of voglibose monotherapy or add-on therapy, which significantly reduced HDA1C, FBG, and PPBG in patients with T2DM. Voglibose was most commonly added with metformin and sulfonylureas. It was well-tolerated by the participants with T2DM without significant weight change. The efficacy of voglibose in a large population with T2DM seen in this study is the real-world reflection of the results obtained in randomized trials.
1. Pradeepa R, Mohan V. Prevalence of type 2 diabetes and its complications in India and economic costs to the nation. Eur Clin Nutr. 2017;71:816-24.

2. Schort RJ. Targeting plasma glucose: preprandial versus postprandial. Clin Diab. 2004;22:169-72.

3. Riddle MC. Basal glucose can be controlled, but the prandial problem persists-it's the next target! Diabetes Care 2017:40:291-300.

4. Vichayanrat A, Ploybutr S, Tunlakit M, et al. Efficacy and safety of voglibose in comparison with acarbose in type 2 diabetic patients. Diabetes Res Clin Pract. 2002;55:99-103.

5. Lee MY, Choi DS, Lee MK, et al. Comparison of acarbose and voglibose in diabetes patients who are inadequately controlled with basal insulin treatment: randomized, parallel, open-label, active-controlled study. J Korean Med Sci. 2014:29:90-7.

6. Blonde L, Khunti K, Harris SB, et al. Interpretation and impact of real-world clinical data for the practicing clinician. Adv Ther 2018:35:1763-74.

7. Riyaz M, Imran S, Joseph NK, Saba M. Role of voglibose in prevention of type 2 diabetes in established case of impaired glucose tolerance: an observation study. Int J Res Med Sci. 2015;3:1453-6.

8. Lodhi MAK, Khatoon AS. Voglibose and its significance in prediabetes: An observational study. Int I Med Health Res. 2019:5:153-4.
9. Torrent Pharmaceuticals Ltd. Prescribing information of Voglitor MD (voglibose) tablet. 2014. Available at: www.torrentian.com/ pisheet/Upload/PI_Sheet/1229.pdf (accessed February 20, 2020)

10. Chen $X$, Zheng Y, Shen Y. Voglibose (Basen, AO-128), one of the most important alpha-glucosidase inhibitors. Curr Med Chem. 2006;13:109-16

11. Santra $D$, Totade $S$. Prescribing trends of antidiabetic fixed dose combinations in a rural tertiary care teaching hospital in central India: an observational, cross-sectional study. Research Journal of Pharmacology and Pharmacodynamics. 2015;7:19.

12. Kumar L, Gupta SK, Prakash A. Assessment of the prescription pattern of anti-diabetic drugs in type-2 diabetes mellitus participants. The Pharma Innovation. 2018;7:392-4.

13. Aparna A, Latha $S$, Nagariun $G$, et al. A study on drug utilization pattern and effectiveness of oral hypoglycemic agents in diabetes mellitus. PharmaTutor. 2015:3:31-7.

14. Ismail D, Deshmukh S. Comparative study of effect of alpha glucosidase inhibitors-miglitol, acarbose and voglibose on postprandial hyperglycaemia and glycosylated hemoglobin in type-2 diabetes mellitus. Int J Pharm Bio Sci. 2012;3:337-43.

15. Jindal A, Jindal M, Kaur M, et al. Efficacy and safety of voglibose as an add-on triple drug in participants of type two diabetes mellitus uncontrolled with glimepiride and metformin in Punjabi population. Indian Journal of Basic and Applied Medical Research. 2014:3:111-6.
16. Talaviya PA Saboo BD, Dodiya HG et al Retrospective comparison of voglibose or acarbose as an add-on therapy to sulfonylureas in Western Indian participants with uncontrolled overweight/obese type 2 diabetes. Diabetes Metab Syndr. 2016;10:88-91.

17. Rao C, Faruqui AA. Efficacy and safety of oral triple drug combination (voglibose, glimepiride and metformin) in the management of type 2 diabetes mellitus. Journal of Current Research and Review 2013:5:20.

18. Yamasaki Y, Katakami $\mathrm{N}$, Hayaishi-Okano R, et al. Alpha-glucosidase inhibitor reduces the progression of carotid intima-media thickness. Diabetes Res Clin Pract. 2005;67:204-10.

19. Seino Y, Fujita T, Hiroi S, et al. Alogliptin plus voglibose in Japanese participants with type 2 diabetes: a randomized, double-blind, placebo-controlled trial with an open-label, long-term extension. Curr Med Res Opin. 2011:27(Suppl. 3):21-9.

20. Deb T, Chakrabarty A, Ghosh A. Adverse drug reactions in Type 2 diabetes mellitus patients on oral antidiabetic drugs in a diabetes outpatient department of a tertiary care teaching hospital in the Eastern India. Int J Med Sci Public Health. 2017;6:554-8.

21. Dabhi AS, Bhatt NR, Shah MJ. Voglibose: an alpha glucosidase inhibitor. J Clin Diagn Res. 2013;7:3023-7.

22. Mathieu C, Barnett AH, Brath $\mathrm{H}$, et al. Effectiveness and tolerability of second-line therapy with vildagliptin vs. other oral agents in type 2 diabetes: a real-life worldwide observational study (EDGE). Int J Clin Pract. 2013:67:947-56. 